\title{
Le paysage religieux de la République et de l'Irlande du Nord au début du XXIe siècle
}

\section{Catherine Piola}

\section{(2) OpenEdition \\ 12 Journals}

Édition électronique

URL : http://journals.openedition.org/etudesirlandaises/3869

DOI : 10.4000/etudesirlandaises.3869

ISSN : 2259-8863

\section{Éditeur}

Presses universitaires de Rennes

\section{Édition imprimée}

Date de publication : 20 novembre 2014

Pagination : 17-35

ISBN : 978-2-7535-3559-6

ISSN : 0183-973X

\section{Référence électronique}

Catherine Piola, « Le paysage religieux de la République et de l'rlande du Nord au début du XXIe siècle », Études irlandaises [En ligne], 39-2 | 2014, mis en ligne le 20 novembre 2016, consulté le 19 avril 2019. URL : http://journals.openedition.org/etudesirlandaises/3869 ; DOI : 10.4000/ etudesirlandaises.3869 


\title{
Le paysage religieux de la République et de l'Irlande du Nord au début du XXI ${ }^{\mathrm{e}}$ siècle
}

\author{
Catherine Piola \\ Université Paris-Dauphine
}

\section{Résumé}

Depuis les années 1990, le paysage religieux irlandais de la République et de l'Irlande du Nord s'est modifié sous l'effet de profonds changements démographiques, culturels et idéologiques. Les données recueillies lors des derniers recensements permettent de retracer cette évolution vers une société du $\mathrm{xxI}^{\mathrm{e}}$ siècle où le bi-confessionalisme chrétien est moins présent, laissant la place à une nouvelle et relative diversité des confessions, et où la désaffection religieuse rallie des effectifs de plus en plus élevés, bien que ceux-ci soient encore minoritaires et difficiles à dénombrer avec précision.

Mots clés : religiosité, désaffection religieuse, catholicisme, protestantisme, diversité religieuse

\section{Abstract}

Since the 1990s, the Irish religious landscape in the Republic and Northern Ireland has changed as a result of the deeply-rooted impact of demographic, cultural and ideological developments. The data collected during the most recent censuses show a $21^{\text {st }}$-century society where the two main Christian religions are less prevalent, allowing a new and relative diversity of confessions, as well as growing numbers of non-believers, even though the size of the latter group remains difficult to determine in an accurate manner.

Keywords : religiosity, secularization, Catholicism, Protestantism, religious diversity

Documenter, mesurer et analyser la place de la religion dans une société est un véritable défi en soi. L'étude relève en effet de l'espace institutionnel qui est donné à la religion dans la société ainsi que de la valeur que lui accorde chaque individu. C'est dans la société contemporaine irlandaise de la République et de l'Irlande du Nord que les paragraphes qui suivent tentent de définir l'attachement religieux des individus composant ces deux nations.

Considérée ici comme marqueur indentitaire, l'appartenance religieuse permet de dessiner un profil démographique précis et contrasté de la population irlandaise à la fin de la première décennie du $\mathrm{xxI}^{\mathrm{e}}$ siècle. L'objectif est de cerner la composition des communautés les plus représentées dans les deux sociétés, leurs 
caractéristiques structurelles et identitaires. L'étude doit aussi s'intéresser à ceux qui, résidents dans le pays, étrangers ou non, se déclarent d'une dénomination religieuse plus mineure tout comme enfin à ceux qui n'ont aucune affiliation religieuse.

L'élaboration de ce profil repose essentiellement sur les données recueillies lors du dernier recensement en date, en 2011, tout en s'appuyant sur les précédentes énumérations décennales ${ }^{1}$. Ce choix nécessite quelques remarques liminaires quant au recueil et à la fiabilité des données.

Le recueil de données relatives à l'identité religieuse de la population soulève des questions quel que soit le pays étudié. Dresser un profil du caractère religieux d'une population à partir des recensements de la population est pourtant plus aisé en Irlande que dans d'autres pays européens. En France ou en Belgique par exemple, les recensements ne dénombrent pas la population selon ce critère, en Allemagne et au Royaume-Uni encore, les données ne sont collectées que depuis peu. Or en Irlande, le critère de l'appartenance religieuse est renseigné depuis le milieu du XIx ${ }^{\mathrm{e}}$ siècle et l'Irlande du Nord, à sa création, a maintenu l'inclusion des données dans ses recensements successifs, contrairement à ce qui a longtemps été fait dans le Royaume-Uni, qui n'a introduit ce critère que depuis 2001.

Dans la République, le recueil de données a longtemps été réduit à la simple déclaration de l'appartenance à une religion; seuls le catholicisme, quelques dénominations protestantes et le judaïsme étaient présentées au public, et ceci jusqu'au recensement de 1961 .

La représentativité des catholiques et protestants était telle, oscillant entre 99,7 et 99,3\% jusqu’à la fin des années 1960, que le bureau des statistiques irlandais jugeait suffisante la catégorie désignée sous le nom de " autre » et regroupant pêlemêle les personnes sans religion, celles se déclarant d'une autre confession, chrétienne ou non, et celles ne souhaitant pas renseigner cet item. Depuis le recensement de 1961, et trente ans durant, ces trois catégories ont fait l'objet de calculs statistiques différenciés, la catégorie " autres religions " n’étant pas plus détaillée. Depuis le recensement de 1991 et sous l'effet d'une diversité religieuse de plus en plus visible, la publication des recensements offre un panorama relativement complet des religions minoritaires présentes dans la République.

En Irlande du Nord, le phénomène est identique : le nombre infime de personnes ne se déclarant ni protestant ni catholique semble avoir justifié longtemps une catégorie fourre-tout intitulée " autre ». De plus, il faut noter que les recensements de 1971 et 1981 ont été perturbés par le climat politique, faussant le recueil des données de l'item « religion » en particulier. Depuis le recensement

1. Les recensements ont lieu tous les cinq ans dans la République mais les questions relatives à la religion ne figurent que dans un recensement sur deux. 
de 1991, plus fiable que les deux précédents, la réponse "item non renseigné » est traitée comme catégorie à part entière, et celle des "autres dénominations " est devenue plus précise à partir de 2001. C'est aussi en 2001 que la distinction a été introduite entre religion pratiquée et religion liée à l'identité familiale dans laquelle la personne interrogée a grandi.

La fiabilité des données des recensements repose avant tout sur leur exhaustivité et la richesse que le croisement des données offre, pour un même recensement comme d'un recensement à l'autre. En 2011, les énumérations de la population fournissent une masse de données sur la religion qui résulte d'un processus de recueil de données plus fin à mesure que les appartenances et les pratiques se sont diversifiées.

Si la fiabilité des recensements pose moins problème que celle de sondages sur des échantillons limités en nombre par exemple, il est vrai que ces données ont leurs limites. L'information est en effet recueillie sur le principe de l'auto-déclaration, non obligatoire qui plus est. Telles qu'elles sont collectées par les agents recenseurs, les données ne peuvent rendre compte de la foi, des pratiques liées à l'appartenance comme l'assiduité aux services, du recours à la prière ou à la confession, du respect ou du rejet des préceptes par exemple.

Afin de compléter le profil démographique qui suit, les sondages nationaux ou européens constituent une source de données qui peut être consultée, sur les questions de la désaffection religieuse ou de la spiritualité notamment. Quelques études européennes, comme l'Eurobaromètre de 2010, fournissent un complément d'information qualitatif vers lequel le lecteur pourra se tourner afin d'en savoir plus sur le sentiment religieux.

Pour lors, l'analyse des recensements successifs fait émerger un profil religieux des Irlandais qui présente trois traits distinctifs et néanmoins liés. On peut en effet dans un premier temps questionner la place du catholicisme et du protestantisme dans l'île avant d'observer la montée relative d'autres confessions, chrétiennes ou non, et enfin se pencher sur la question du rejet ou de l'absence de religion.

\section{Une écrasante et durable dualité catholique/protestante}

Situons pour commencer les deux religions majoritaires, catholique et protestante, dans une perspective historique qui permet de comprendre pourquoi on a si longtemps associé d'une part la République à un fort catholicisme et en quoi, par ailleurs, la population de l'Irlande du Nord a longtemps été déchirée entre les deux religions chrétiennes majoritaires. 
Tableau 1 : Effectifs catholiques et protestants et part de la population totale en République d'Irlande et en Irlande du Nord, de 1911 à 2011 (* en millions) (Source: Volume 13, Census 2006, p. 9 et This is Ireland 1, 2011, p. 104, et Northern Ireland Census 1911, 1926, 1961, 1981, 1991 et 2011 nisra. gov.uk)

\begin{tabular}{|c|c|c|c|c|c|c|}
\hline & 1911 & 1926 & 1961 & 1981 & 1991 & 2011 \\
\hline \multicolumn{7}{|c|}{ République d'Irlande } \\
\hline $\begin{array}{l}\text { Catholiques* } \\
\%\end{array}$ & $\begin{array}{c}2,812 \\
89,5\end{array}$ & $\begin{array}{c}2,751 \\
92,5\end{array}$ & $\begin{array}{c}2,673 \\
94,8\end{array}$ & $\begin{array}{c}3,204 \\
93\end{array}$ & $\begin{array}{c}3,228 \\
91,5\end{array}$ & $\begin{array}{c}3,861 \\
84,1\end{array}$ \\
\hline $\begin{array}{l}\text { Protestants } \\
\%\end{array}$ & $\begin{array}{c}249535 \\
9,8\end{array}$ & $\begin{array}{c}207307 \\
6,9\end{array}$ & $\begin{array}{c}129645 \\
4,5\end{array}$ & $\begin{array}{c}115411 \\
3,2\end{array}$ & $\begin{array}{c}107423 \\
2,9\end{array}$ & $\begin{array}{c}165807 \\
3,5\end{array}$ \\
\hline \multicolumn{7}{|c|}{ Irlande du Nord } \\
\hline $\begin{array}{l}\text { Catholiques } \\
\%\end{array}$ & $\begin{array}{c}430161 \\
34,4\end{array}$ & $\begin{array}{c}420428 \\
33,5\end{array}$ & $\begin{array}{c}497547 \\
34,9\end{array}$ & $\begin{array}{c}414532 \\
28\end{array}$ & $\begin{array}{c}605639 \\
38,4\end{array}$ & $\begin{array}{c}738033 \\
40,8\end{array}$ \\
\hline $\begin{array}{l}\text { Protestants } \\
\%\end{array}$ & $\begin{array}{c}768056 \\
61,4\end{array}$ & $\begin{array}{c}781652 \\
62,2\end{array}$ & $\begin{array}{c}829778 \\
58,2\end{array}$ & $\begin{array}{c}792843 \\
53,5\end{array}$ & $\begin{array}{c}763214 \\
48,3\end{array}$ & $\begin{array}{c}752555 \\
41,6\end{array}$ \\
\hline
\end{tabular}

La part de la religion catholique au lendemain de la création de l'État Libre s'est étendue au cours des quarante années qui ont suivi, soutenue par l'idéologie de la constitution de 1937 et alors même que le nombre de catholiques dans l'Irlande de la fin du XIx ${ }^{e}$ siècle était légèrement moindre ${ }^{2}$, le nombre d'Anglais, protestants, installés dans le pays étant alors plus élevé3. Le document révèle parallèlement le recul du nombre et de la proportion de membres déclarant leur appartenance au protestantisme, qui reste néanmoins numériquement la seconde minorité religieuse dans la République.

Le catholicisme occupe encore au début des années 2010 la position de religion de la majorité de la population de la République, quand bien même la place des rites catholiques, comme la présence à la messe dominicale ou la cérémonie du baptême, sont en net recul si l'on en croit les études européennes. Les transformations de pratiques sociales comme le mariage religieux ou l'organisation maritale et familiale par exemple, indiquent aussi une prise de distance par rapport aux préceptes catholiques qui n'est, de toute évidence, pas incompatible avec l'identité catholique dont se revendiquent encore $84 \%$ des Irlandais. Il s'agit d'une communauté qui a repris une croissance en nombre depuis la fin des années 1980 mais dont la part proportionnellement au reste de la population est en déclin, tout comme les effectifs et la part des protestants dans la République.

Dans la République, le recul du catholicisme n'est en effet pas dû à la montée du protestantisme puisqu'il a peu varié proportionnellement depuis les années 1970. Bien qu'étant demeurée seconde confession en nombre de pratiquants, le

2. On relève $89 \%$ de catholiques lors du recensement de 1891.

3. On estime que la création de l'État Libre a motivé le départ de près de 100000 personnes, quasi-exclusivement des protestants. 
protestantisme dont l'Église d'Irlande demeure la dénomination la plus représentée a vu ses effectifs se réduire depuis la création de la nation jusqu'aux années 1990 , passant de près de $8 \%$ à moins de $3 \%$ de la population totale. Le nombre croissant de l'ensemble des protestants depuis ne permet que de maintenir dans la République une proportion d'à peine plus de 3,5\% de l'ensemble de la population. Les différentes affiliations de cette branche du christianisme lui donnent une moins grande unité ou visibilité publique.

Historiquement, le nombre de membres des deux religions chrétiennes en Irlande du Nord a été plus équitablement partagé que dans la République. L'apparent déclin du nombre de catholiques dans les années 1980 est sans doute lié à la situation politique de la période; ce déclin peut en partie s'expliquer aussi par un plus grand nombre de personnes refusant de déclarer leur affiliation religieuse, pour des raisons politiques ou plus personnelles. Le manque de précision sur cet item du recensement ne permet pas de confirmer la validité de cette hypothèse.

Afin d'analyser les causes des fluctuations des deux confessions, de comprendre en quoi elles subissent les effets de facteurs démographiques ou sociétaux, les paragraphes suivant réunissent les principales caractéristiques de chacune des deux communautés en 2011.

Les catholiques recensés dans la République sont très majoritairement irlandais, à $92 \%$. Leur représentation par classes d'âges et dans les différentes catégories professionnelles reflète celle de l'ensemble de la population tant leur nombre domine au sein de celle-ci. Ainsi, les catholiques de moins de 30 ans représentent $40 \%$ de l'ensemble des catholiques, les protestants du même âge sont $37 \%$ du total des protestants.

Un tiers des catholiques irlandais appartient aux catégories professionnelles les plus élevées, un autre aux catégories des travailleurs manuels, le dernier tiers étant occupé à des postes intermédiaires.

En Irlande du Nord, les catholiques de moins de 30 ans forment un groupe de population plus jeune que celui des protestants du même âge, comptant respectivement pour $44 \%$ et $34 \%$ de leur communauté. Les catholiques nord irlandais sont aussi socialement marqués. Ils sont en effet moins nombreux à occuper des postes à responsabilité, moins bien qualifiés et plus nombreux proportionnellement à être sans emploi.

Sur le territoire, la répartition des catholiques est moins marquée dans la République qu'en Irlande du Nord. On note cependant une très relative faiblesse du catholicisme dans la région de Dublin, où le moindre attachement religieux des citadins couplé à la diversité d'autres confessions dans la capitale et sa région en sont principalement les causes. À mesure que l'on s'éloigne de la capitale, vers le centre et l'ouest, la proportion de catholiques augmente, Offaly étant le comté où, proportionnellement, le nombre de personnes se déclarant de cette religion est le plus élevé. 
Sans surprise aussi, les comtés bordant l'Irlande du Nord sont moins marqués par la domination catholique, la proportion de protestants y étant plus grande. Les variations d'un comté à l'autre, d'une région à l'autre sont aussi très liées au cadre de vie : en milieu citadin, l'identité religieuse joue un rôle plus secondaire qu'en milieu rural où les populations sont en moyenne plus âgées, l'isolement est plus grand, les communautés plus soudées, notamment par les cérémonies et rituels religieux qui ont encore une fonction sociale importante. Pour les femmes, l'attachement religieux est plus marqué; ceci vaut pour les catholiques comme pour la plupart des autres religions présentes sur le territoire.

La répartition territoriale des catholiques en Irlande du Nord est et demeure marquée, selon les comtés, les circonscriptions électorales et les quartiers des villes du pays. Les comtés bordant la République, au sud et à l'ouest, sont majoritairement catholiques, alors que la région est du pays est majoritairement peuplée de protestants. Contrairement à ce qui existe dans la République, le brassage territorial est quasi-inexistant et le sens de l'appartenance religieuse reste fortement associé à un espace strictement délimité. La capitale est un exemple à la fois de divisions communautaires, avec ses quartiers nord et est très majoritairement protestants, et ceux de l'ouest qui sont catholiques.

Enfin en terme d'identité nationale, parmi la minorité (7\%) d'étrangers de confession catholique résidant dans la République, notons que la plus grande partie est européenne, 82 \% d'entre eux. En 2011, les Polonais et les Lituaniens constituaient plus de la moitié de ce groupe et, avec les catholiques originaires du reste de l'Europe de l'Est (Tchèques, Hongrois, Lettons, Roumains et Slovaques), ils représentaient alors $68 \%$ des catholiques étrangers. En Irlande du Nord, la montée du catholicisme a été renforcée par la présence de résidents non-nationaux, Polonais et Lituaniens, formant les deux minorités nationales numériquement les plus présentes.

Ces données montrent que la domination de la religion catholique en Irlande au début du XXI ${ }^{\mathrm{e}}$ siècle a été renforcée par la forte présence de citoyens de l'Europe de l'Est. Ils sont environ 160000 catholiques dans la République et sans eux, la part de cette confession serait tombée à $81 \%$ dans l'ensemble de la population du pays. On comprend alors les encouragements institutionnels pour organiser la venue de travailleurs étrangers originaires de cette partie de l'Europe lorsque les besoins en main-d'œuvre du Tigre Celtique étaient pressants. Le maintien de l'identité catholique du pays s'en trouvait assuré et la relative désaffection des Irlandais compensée $e^{4}$.

\footnotetext{
4. Une Enquête de 2005 de l'organisation ARK menée par des universitaires de Dublin et Belfast montrait la chute de la fréquentation des messes dominicales depuis les années 1970. Voir ARK 2005, Religion in Ireland : no longer an exception?, Maire Nic Ghiolla Phadraig.
} 
Dans la République, le protestantisme demeure en 2011 la seconde confession par le nombre de ses pratiquants, deux tiers d'entre eux étant irlandais. La communauté des étrangers de confession protestante est pour moitié constituée de ressortissants du Royaume-Uni. Comme en Irlande du Nord, il s'agit d'un groupe en moyenne moins jeune que celui des catholiques. Dans la République et en Irlande du Nord respectivement, $33 \%$ et $39,4 \%$ des protestants ont plus de 50 ans, alors qu'ils ne sont que $28 \%$ dans les mêmes âges chez les catholiques. Ils sont plus nombreux que les catholiques dans les catégories professionnelles plus élevées : $40 \%$ des actifs protestants occupent des professions à responsabilité pour $32 \%$ des catholiques, et ceci bien qu'aucun critère religieux ne participe à la sélection des étudiants des différentes universités des deux pays par exemple, ou encore que la discrimination religieuse soit punie par la loi.

En 2011, les protestants représentent 3,5\% de la population totale de la République, proportion légèrement plus élevée dans les comtés de Donegal et Sligo $(4 \%)$ et dans celui de Cavan $(6,2 \%)$. Contrairement à ce qui a été décrit en Irlande du Nord, la co-existence des deux confessions n'est pas territoriale, bien que des poches à plus forte population protestante existent dans certains milieux urbains, ainsi historiquement dans l'ancienne English Town, aujourd'hui Dun Laoghaire ou encore à West Cork où de nombreux Britanniques possèdent une résidence secondaire.

Près de trois quarts des protestants vivant dans la République sont irlandais, le quart restant étant principalement constitué d'Européens (83 \%) et, très majoritairement des ressortissants britanniques auxquels sont venus se joindre, entre 2006 et 2011, des Lituaniens. Notons enfin que parmi les membres étrangers de l'Église d'Irlande, quelque $8 \%$ sont d'origine africaine.

Les protestants constituent un groupe confessionnel moins unitaire que les catholiques. Le maintien de ses effectifs, plus que sa très modeste croissance depuis 2006, lui vaut encore aujourd'hui le rang de première minorité religieuse de la République.

Confession majoritaire de l'Irlande du Nord, le protestantisme est depuis 2011 la religion de moins de la moitié des résidents de l'Irlande du Nord, soit 48,4 \% toutes dénominations du protestantisme confondues, alors qu'il était encore de $53 \%$ en 2001. Au moins trois facteurs sont liés à ce recul : la structure par âge de la population protestante, le dynamisme démographique moindre la caractérisant et qui découle aussi du vieillissement de la population et, en partie sans doute aussi, la désaffection du sentiment religieux qui caractérise les sociétés occidentales, dont le Royaume-Uni.

L'antagonisme catholique/protestant, peu visible dans la République, est identitairement toujours manifeste en Irlande du Nord. La progressive montée en nombre des catholiques, à Belfast par exemple où ils sont devenus majoritaires en 
20115 , ne réduit pas les tensions qui existent depuis de nombreuses décennies. La question de la mixité territoriale demeure au niveau local comme à celui de la nation.

Le recul du catholicisme dans la République, ralenti depuis 2004 par l'arrivée en nombre de catholiques, d'Europe de l'Est en particulier, et la montée de celui de l'Irlande du Nord attestent des évolutions marquantes en cours. Cependant, outre les deux religions majoritaires, il est nécessaire de se tourner vers d'autres dénominations, chrétiennes et non chrétiennes, pour mieux comprendre les transformations en cours du paysage religieux.

\section{Un paysage religieux de plus en plus varié}

En République comme en Irlande du Nord, la caractéristique qui marque l'évolution de la vie religieuse au cours des trente dernières années est sa plus grande diversité. En effet, restées jusque dans les années 1980 très minoritaires, car représentant un nombre total de pratiquants de l'ordre de 0,1 à $0,3 \%$ de la population, les confessions autres que le catholicisme et les protestantismes ont depuis pris une place grandissante dans le paysage religieux, comme en témoignent les deux documents suivants.

La diversité du paysage religieux irlandais, chrétien et non chrétien, est manifeste en 2011. Islam et orthodoxie sont les deux confessions allogènes les plus visibles au sein du paysage confessionnel de la République et de l'Irlande du Nord. Dans les paragraphes ci-dessous, un portrait de ces deux communautés est proposé, précédé de celui des fidèles du judaïsme; ils sont suivis de commentaires relatifs aux autres dénominations.

Bien que très minoritaire aujourd'hui, le judaïsme est une religion dont l'histoire en Irlande est plus ancienne que celle des autres religions non-chrétiennes du paysage confessionnel d'aujourd'hui. Certains documents attestent de sa présence dans l'île dès le XIII ${ }^{e}$ siècle; il ne s'agissait alors que de quelques individus, remarqués pour leurs contributions à la vie politique et économique du pays. La première synagogue est construite à Dublin en 1660, à Belfast, en 1870, et dans les deux cas pour une colonie restreinte de pratiquants. À partir des années 1870, l'arrivée d'immigrés juifs est avérée; certains fuyaient les pogroms, d'autres étaient des migrants économiques, parmi eux certains en partance pour les États-Unis. Nombre d'entre eux étaient issus de l'actuelle Lituanie et se sont regroupés dans les plus grandes villes du pays, Cork, Limerick, Belfast, et surtout à Dublin dans le quartier South Circular Road du centre ville, rapidement surnommé "Petite

5. Le recensement de 2011 fait état de $49 \%$ de Catholiques à Belfast et dans sa banlieue, soit une augmentation de $4 \%$ depuis 2001 alors que la communauté protestante, représentant 42,3\%, y a reculé de $12 \%$ depuis 2001. 
Tableau 2 : Dénominations religieuses dans la République en 1971, 1991 et 2011 (Source : Volume 13, Census 2006, p. 9, Census 2011, Religion, Ethnicity and Irish Travellers, p. 47, et This is Ireland 1, 2011, p. 104)

\begin{tabular}{|c|c|c|c|}
\hline & 1971 & 1991 & 2011 \\
\hline & \multicolumn{3}{|c|}{ Religions majoritaires } \\
\hline & Total & Total & Total \\
\hline Catholiques (en millions) & 2,795 & 3,232 & 3,861 \\
\hline \multirow[t]{2}{*}{ Protestants Église d'Irlande et autres } & $97739+21298$ & $89187+25766$ & $129039+58866$ \\
\hline & \multicolumn{3}{|c|}{ Religions minoritaires } \\
\hline Effectifs totaux & 6887 & 33989 & 196273 \\
\hline Part de la population totale & $0,2 \%$ & $1 \%$ & $4,7 \%$ \\
\hline Musulmans & & 3873 & 49204 \\
\hline Orthodoxes \& Coptes & & 358 & 45223 \\
\hline Autres chrétiens & & 16329 & 41161 \\
\hline Hindou & & 953 & 10688 \\
\hline Bouddhistes & & 986 & 8703 \\
\hline Témoins de Jehovah & & 3393 & 6149 \\
\hline Luthériens & & 1010 & 5683 \\
\hline Evangéliques & & 1075 & 4188 \\
\hline Juifs & 2633 & 1581 & 1984 \\
\hline Panthéistes & & 202 & 1940 \\
\hline Mormons & & 853 & 1284 \\
\hline Quakers & & 749 & 925 \\
\hline Baha’i & & 430 & 520 \\
\hline Autres religions & 4254 & 2197 & 18621 \\
\hline
\end{tabular}

Jérusalem ». Ces Litvaks constituaient à l'époque la plus importante communauté étrangère du pays qui s'est intégrée peu à peu au tissu économique du pays, principalement par le biais des activités préindustrielles de colporteurs et d'usuriers et donc plus dans l'actuelle République que dans le Nord du pays ${ }^{6}$. Leur essor, en partie dû à une intégration sociale réussie, est rendu visible par une participation à la vie politique et culturelle dans laquelle demeurent toutes les valeurs propres à leur communauté. On compte environ quatre mille membres au milieu du $\mathrm{Xx}^{\mathrm{e}}$ siècle mais la communauté n'a cessé de s'étioler depuis. Ce déclin est lié à un ensemble de facteurs dont le vieillissement du groupe et le départ de nombre de ses plus jeunes membres, à la création de l'État d'Israël et, au cours des décennies suivantes, vers les États-Unis pour des raisons économiques. Aujourd'hui la République compte quatre synagogues, trois à Dublin et une à Cork. Belfast

6. Au début du $\mathrm{xx}^{\mathrm{e}}$ siècle, on dénombre moins de 800 membres de la confession judaïque en Irlande du Nord et environ 3700 en République. 
Tableau 3 : Dénominations religieuses en Irlande du Nord en 1971, 1991 et 2011 (Source : Northern Ireland Census 1971 Religion, p. 16, et Northern Ireland Census 1991 Religion, p. 18, et Table

DC2118NI : Religion by sex, nisra.gov.uk)

* dénominations majoritaires du protestantisme

(Presbytériens, Église d'Irlande, Méthodistes, Baptistes, Brethren, Église d'Angleterre, Pentecôtistes)

\begin{tabular}{|c|c|c|c|}
\hline & 1971 & 1991 & 2011 \\
\hline & \multicolumn{3}{|c|}{ Religions majoritaires } \\
\hline & Total & Total & Total \\
\hline Catholiques & 477919 & 605639 & 738033 \\
\hline \multirow[t]{2}{*}{ Protestants* } & 879135 & 763214 & 752555 \\
\hline & \multicolumn{3}{|c|}{ Religions minoritaires } \\
\hline Effectifs totaux & 19781 & 32288 & 36620 \\
\hline$\%$ de la population totale & $1,7 \%$ & $2,2 \%$ & $2 \%$ \\
\hline Musulmans & 275 & 972 & 3832 \\
\hline Orthodoxes \& Coptes & 51 & 72 & 1988 \\
\hline Hindou & 651 & 742 & 2382 \\
\hline Bouddhistes & 67 & 270 & 1046 \\
\hline Témoins de Jehovah & 1041 & 2121 & 1728 \\
\hline Luthériens & 261 & 123 & 294 \\
\hline Évangéliques & 589 & 730 & 2719 \\
\hline Juifs & 959 & 410 & 335 \\
\hline Mormons & 975 & 1437 & 1236 \\
\hline Quakers & 1019 & 804 & 679 \\
\hline Sikhs & 113 & 157 & 216 \\
\hline Baha’i & 65 & 319 & 238 \\
\hline Autres religions & 13715 & 24131 & 19771 \\
\hline
\end{tabular}

possède aussi sa synagogue pour une petite centaine de membres. Les familles Briscoe et Herzog ou Wolff restent associées à la vie politique et économique de l'île. La communauté juive irlandaise, pourtant historiquement la plus ancienne, est devenue très minoritaire dans le paysage religieux du pays qui a vu d'autres confessions s'établir dans la seconde moitié du $\mathrm{xx}^{\mathrm{e}}$ siècle.

C'est le cas de l'islam, dans la République, qui, d'une présence très minoritaire due à quelques étudiants arrivés dans les années 1950, est devenu la troisième confession après le catholicisme et le protestantisme, et la première confession non chrétienne en nombre de pratiquants. S’il est vrai que les musulmans étaient encore relativement peu nombreux au début des années 1990, 3873 énumérés lors du recensement de 1991, le nombre de réfugiés serbes, kosovars et somaliens ainsi que celui d'étudiants venus de Malaisie, du Pakistan ou du Proche-Orient ont participé à la croissance de cette communauté au cours des années 1990. Par 
la suite, l'arrivée de travailleurs qualifiés, des Philippines notamment, a encore accru les effectifs. La communauté s'est organisée autour de sociétés, fondées sur une participation associative à Cork, Galway ou Limerick, à partir des années 1980. Elles permettent de réunir les membres de cette confession qui ne partagent souvent ni la même culture ni la même langue.

En 2002, la République comptait 19147 musulmans, soit 0,4 \% de la population totale du pays, 32539 en 2006 et 49204 en 2011, soit respectivement 0,7 et $1 \%$ de la population totale. Les femmes musulmanes sont minoritaires, leurs effectifs (21000) représentent $42 \%$ de l'ensemble du groupe. Les musulmans sont le second groupe confessionnel ayant la plus forte croissance, après les orthodoxes. Cet essor doit être considéré toutes proportions gardées car leurs effectifs restent modestes, notamment comparativement à ceux des deux religions majoritaires.

La moitié de la communauté islamique de la République réside à Dublin et dans sa banlieue; c'est dans un quartier sud de la capitale qu'un premier lieu de culte a été établi au milieu des années 1970 alors que la première société islamique avait été créée à la fin des années 1950. Située dans une ancienne église presbytérienne de ce même quartier, la première mosquée du pays a été inaugurée en 1983, et la première école publique et musulmane dix ans plus tard. Depuis, cette discrète communauté n'a cessé de croître en nombre et en importance dans la vie du pays. L'élection au Parlement d'un de ses membres ${ }^{7}$, en 1992, constitue un pas en avant en matière de visibilité et d'implication dans la vie du pays. Il s'agit d'une communauté qui demeure diverse car constituée à environ $70 \%$ d'étrangers dont $9 \%$ d'Européens, $18,5 \%$ d'Africains et 32,4\% d'Asiatiques. Pourtant, les musulmans irlandais constituent une part grandissante de l'ensemble de la communauté islamique. De 2006 à 2011, le nombre d'Irlandais musulmans a quasiment doublé, passant de 9700 membres à 18200 , alors que la population musulmane étrangère n'était passée que de 21600 à 29100 membres. Ces chiffres montrent que l'islam est plus ancré dans la société irlandaise que d'autres confessions non traditionnelles qui reposent sur un vivier de pratiquants majoritairement étrangers. Chez les orthodoxes notamment, la part des fidèles de nationalité irlandaise est nettement plus réduite. Chez les musulmans, on peut penser que la communauté prendra plus d'ampleur encore au cours des années à venir du fait de l'âge relativement peu élevé des membres du groupe. En effet, trois musulmans sur dix sont âgés de moins de quatorze ans et les trois quarts des musulmans d'Irlande ont moins de trente-cinq ans en 2011. La taille de cette jeune population a motivé la création d'une école publique à Dublin, où étaient inscrits près de 200 enfants en 2011 et où l'État contribue au financement des activités éducatives, à l'exception de l'enseignement de la religion.

7. Il s'agit du parlementaire Moosajee Bhamjee élu au Parlement irlandais de 1992 à 1997. 


\section{Catherine Piola}

Les musulmans d'Irlande du Nord forment une communauté majoritairement composée d'étrangers arrivés en nombre au cours des dix dernières années et ne représentant que $0,2 \%$ de la population totale et $10,4 \%$ des effectifs des religions minoritaires, c'est-à-dire une minorité moins présente que dans la population de la République où ils représentent désormais $1 \%$ de la population totale et un quart des effectifs des religions minoritaires. Il s'agit pourtant de la plus grande minorité non chrétienne d'Irlande du Nord qui, selon le centre musulman de Belfast pourrait atteindre les 10000 membres et qui pourtant n'a pas de lieu de culte pouvant accueillir un grand nombre de fidèles. Au début des années 2000, le projet de construction d'un centre musulman doté d'une mosquée à Craigavon, dans le comté d'Armagh, a été abandonné suite aux plaintes de riverains et à des attaques racistes dont ont été victimes certains membres de la communauté, l'imam et sa famille en particulier.

À ce jour, ni Belfast, qui réunit le plus grand nombre de pratiquants, ni une autre ville du pays ne sont prêtes à accueillir les musulmans d'Irlande du Nord afin qu'ils puissent implanter un espace culturel et religieux correspondant aux besoins de la communauté.

Dans la République, les effectifs et les besoins sont tels que le centre du sud de la capitale, ouvert en 1996 à Clonskeagh, ne répond déjà plus aux besoins de la communauté musulmane du Grand Dublin. En avril 2013, au nord de Dublin, à Clongriffin, le projet de construction d'un nouveau centre islamique comprenant une mosquée semble prendre forme. Accueilli par certains comme un moyen de relancer le secteur du bâtiment du fait de l'ampleur du projet qui comprend un centre culturel, un lieu de culte, des écoles primaires et secondaires et des logements, il suscite aussi pour la première fois dans la République la crainte d'une intégration plus difficile des fidèles musulmans ainsi refoulés et concentrés aux confins de la zone urbaine dublinoise.

Il faut souligner que, jusqu’à présent, les questions du port de signes distinctifs de la religion, de la pratique des prières quotidiennes, du ramadan ou encore celles associées aux contraintes alimentaires n’ont pas déclenché dans la République les prises de position qu'elles ont suscitées sur le continent. En 2012, un colloque international consacré à l'islam en Irlande s'est tenue à l'université de Cork. La volonté y était affichée d'ouvrir un champ d'investigation en sciences sociales sur des sujets de réflexion comme l'existence de l'islam dans un pays majoritairement chrétien, les secondes générations de musulmans en Irlande, la conversion à l'islam et les identités multiples. Il s'agit sans aucun doute d'une manière constructive de prendre conscience des défis que pose la présence de cultures et de cultes minoritaires dans un pays encore très marqué institutionnellement par les religions chrétiennes dominantes. 
Les orthodoxes irlandais participent de cette diversité. Bien que chrétiens, ils ne sont présents dans l'île que depuis quelques décennies. En effet, bien qu'un petit nombre de Russes blancs se soient installés dans le pays peu après la révolution russe, disséminés dans plusieurs régions, ils n’ont jamais constitué une minorité religieuse organisée et visible. La première communauté orthodoxe officielle était grecque, établie dans la capitale de la République en 1981 à St Mary's Church puis à St Peter and Paul en 1986, et elle réunissait environ 350 membres, des ressortissants grecs principalement. En l'espace de deux décennies, l'orthodoxie est passée au rang de seconde confession chrétienne non traditionnelle (ni catholique ni protestante) en nombre de pratiquants. En 2002, ils étaient déjà près de trente fois plus nombreux qu'en 1991, soit un accroissement annuel de l'ordre de 900 membres, dont le plus grand nombre $(85 \%)$ n'était pas irlandais. Sur la courte période de 2002 à 2006, leurs effectifs ont doublé, passant de 10000 à 20000 et, de 2006 à 2011, ils ont de nouveau doublé et dépassent aujourd'hui les 45000 membres dans la République, soit près de $1 \%$ de l'ensemble de la population.

En Irlande du Nord, leur nombre est resté très minoritaire jusqu'au milieu des années 2000 et l'essor de la communauté est nettement lié à l'arrivée d'Européens de l'Est suite à l'élargissement de l'Union Européenne en 2004. En effet, la politique non restrictive de la République et dans une moindre mesure de l'Irlande du Nord en matière de flux migratoires et le dynamisme économique de l'époque expliquent cette fulgurante progression. Ainsi, $65 \%$ des orthodoxes de la République sont des ressortissants de l'Union Européenne, arrivés à partir de 2004, Roumains et Lettons pour respectivement $51 \%$ et $24 \%$ de ces immigrants (voir tableau 4). Un peu plus d'un quart des autres orthodoxes vivant en Irlande est issu du reste de l'Europe, de Russie et d'Ukraine. Enfin, parmi les quelques 3000 orthodoxes venus d'Asie, près de la moitié sont Indiens.

Le groupe est ainsi constitué, majoritairement de non-Irlandais puisqu'à peine un orthodoxe sur cinq en Irlande est de nationalité irlandaise. Plus de la moitié des orthodoxes $(58 \%)$ a entre 25 et 44 ans en 2011 ; ils sont actifs pour la plupart, et sont aussi les moins présents dans les classes d'âge de plus de 55 ans ainsi que chez les jeunes de moins de quatorze ans. Par ailleurs, neuf sur dix sont citadins. Ce profil reflète la plupart des caractéristiques des migrants économiques qui constituent la majeure partie du groupe.

La pratique d'une même religion ne crée pas nécessairement une communauté et dans le cas précis de l'orthodoxie, les différences linguistiques et culturelles, mais aussi de traditions et de rituels rendent le groupe plus disparate que d'autres groupes religieux. Plusieurs branches de l'orthodoxie sont représentées dans la République depuis le début des années 2000 et les services religieux se font souvent dans plusieurs langues : en roumain ou en serbe et en anglais le plus 
souvent. Géographiquement, ils sont près des trois quarts à vivre dans l'Est du pays, Dublin et sa banlieue réunissant plus de la moitié de tous les orthodoxes d'Irlande. Bien que cette concentration dans le Leinster s'accentue depuis 2002, notons qu'ils sont aussi présents dans le sud du pays pour $15 \%$ d'entre eux. Depuis 2006, les régions de Cork et Galway ont un prêtre, qui officie aussi occasionnellement dans des zones plus isolées, comme dans certaines villes moyennes du Kerry ou du centre du pays. En 2010, deux nouvelles congrégations, à Athlone et Drogheda se sont formées, là encore, en réponse aux besoins de nombreux ressortissants qui souhaitent maintenir leur tradition dans un contexte où le catholicisme est dominant.

On note ainsi que la chrétienté n'est plus exclusivement catholique ou anglicane (Église d'Irlande) dans la République du Xxi siècle : les autres religions chrétiennes réunissent, en 2011, quelque 150000 membres, soit trois fois plus que vingt ans auparavant. Le nombre croissant de pentecôtistes par exemple est majoritairement dû à la présence d'étrangers, principalement venus d'Afrique. Toutes les dénominations, à l'exception du méthodisme, ont renforcé leur présence au cours de cette période avec de plus ou moins fortes variations.

Parmi les religions non chrétiennes, l'hindouisme et le bouddhisme se sont répandus au cours des vingt dernières d'années, passant de moins d'un millier de membres au début des années 1990 à respectivement plus de 10000 et 8000 membres dans la République. Il s'agit, pour plus de la moitié, de ressortissants indiens auxquels se joignent des Britanniques, une partie d'entre eux étant également originaire d'Inde. Enfin, la petite communauté Baha'i, dont les premiers membres sont arrivés au titre de réfugiés en 1985 et dont la plupart sont devenus depuis citoyens irlandais, contribue aussi à la diversité de ce nouveau paysage religieux irlandais.

La catégorie " autres religions " est trop vague pour permettre une analyse détaillée. Là encore, la progression depuis vingt ans est forte, de quelque deux mille à quatorze mille individus dans la République, et les pratiques et les lieux de cultes restent très en marge des confessions les plus reconnues et visibles. En Irlande du Nord, sans doute du fait de la plus grande proximité avec le RoyaumeUni, dès le début des années 1970, la diversité existait, elle demeure relativement élevée étant donnée la taille de la population totale. Le recul récent indique qu'il s'agit d'une diversité religieuse très largement due à la présence d'étrangers qui, pour certains d'entre eux, ne sont pas restés en Irlande du Nord après la crise financière de 2008. Dans l'ensemble de l'île, il s'agit de groupes minoritaires qui contribuent néanmoins, au même titre que les autres, à la grande variété du paysage religieux irlandais du XXI ${ }^{\mathrm{e}}$ siècle et, à ce titre, qui mériteraient un recensement plus fin. La nationalité des personnes recensées éclaire l'analyse de cette nouvelle diversité. Les données ci-dessous relatives à la République montrent 
l'étroit lien entre les communautés religieuses et les différentes origines territoriales de leurs membres.

Tableau 4: Appartenance religieuse et origines nationales ou territoriales dans la République en 2011

(Source : Profile 7, Religion, Ethnicity and Irish Travellers, Census 2011, p. 52)

* Église d'Irlande (+ Presbytériens et Pentecôtistes)

\begin{tabular}{|l|c|c|c|c|c|c|c|c|c|}
\hline & Irlandais & Étrangers & $\begin{array}{c}\text { Royaume- } \\
\text { Uni }\end{array}$ & Reste UE & $\begin{array}{c}\text { Reste } \\
\text { Europe }\end{array}$ & Afrique & Asie & Amérique & Autre \\
\hline Catholiques & 3525573 & 282799 & 49761 & 184066 & 16307 & 41642 & 19420 & 13706 & 3549 \\
\hline Protestants* & $\begin{array}{c}93056 \\
+19868\end{array}$ & $\begin{array}{c}30464 \\
+16493\end{array}$ & $\begin{array}{c}21477 \\
+3802\end{array}$ & $\begin{array}{c}4228 \\
+3814\end{array}$ & $\begin{array}{c}2222 \\
+104\end{array}$ & $\begin{array}{c}2571 \\
+6771\end{array}$ & $\begin{array}{c}783 \\
+713\end{array}$ & $\begin{array}{c}13706 \\
+909\end{array}$ & $\begin{array}{c}563 \\
+339\end{array}$ \\
\hline Musulmans & 18223 & 29143 & 823 & 1445 & 2049 & 8777 & 15376 & 170 & 427 \\
\hline Orthodoxes & 8465 & 34854 & 271 & 22108 & 8901 & 815 & 2299 & 154 & 268 \\
\hline $\begin{array}{l}\text { Autres chré- } \\
\text { tiens }\end{array}$ & 24023 & 15258 & 2112 & 3165 & 300 & 5506 & 2556 & 1325 & 294 \\
\hline Autres & 34867 & 40227 & 6578 & 11865 & 621 & 4967 & 12620 & 2891 & 1907 \\
\hline Sans Religion & 173180 & 82194 & 25620 & 37463 & 1478 & 1154 & 10179 & 4188 & 1907 \\
\hline Non renseignée & 29888 & 12925 & 1795 & 6371 & 458 & 1311 & 1633 & 913 & 433 \\
\hline
\end{tabular}

Les deux premières colonnes du document permettent de juger du rôle joué par les populations étrangères dans la diversité du paysage religieux irlandais d'aujourd'hui. Les membres des églises orthodoxe, musulmane et pentecôtiste sont majoritairement étrangers. Cela vaut, dans une moindre mesure, pour l'ensemble des autres religions non chrétiennes. Par ailleurs, si l'on compare les chiffres de 2011 à ceux de 2006, on s'aperçoit que les variations intercensitaires sont beaucoup plus fortes chez les Irlandais musulmans et orthodoxes par exemple, que chez les étrangers membres de ces églises. L'examen du nombre de conversions religieuses et celui des naturalisations pourraient sans doute fournir des explications à ce phénomène. Ces données n'étant pas disponibles à ce jour, on ne pourra que conclure cette section en soulignant que la plus grande diversité religieuse dans la République au $\mathrm{XxI}^{\mathrm{e}}$ siècle ne repose pas uniquement sur la présence de populations étrangères. Les Irlandais participent aussi à ce renouveau.

Le défi de la République et de l'Irlande du Nord sera de trouver les moyens de permettre à ses citoyens et résidents de vivre en harmonie, voire dans une société où le brassage religieux et culturel existe réellement. Ces questions ne sont pas abordées ici; elles constituent pourtant des sujets d'études révélateurs de la manière dont peut être vécue la nouvelle diversité religieuse. 


\section{Montée inédite de l'absence de religion et de l'athéisme}

Dans la République, le recueil des données relatives à l'absence de religion est demeuré imprécis jusqu'au recensement de 1961; en effet, jusqu’à cette date, la catégorie 'Autres religions' incluait toutes les réponses autres que catholique, protestant, méthodiste, juif ou baptiste. Par conséquent, les personnes sans religion, celles qui ne renseignaient pas l'item ou qui étaient athées, voire agnostiques, étaient comptabilisées dans une catégorie qui représentait, en 1926 comme en 1936, 0,3\% de la population totale de la République.

Tableau 5 : Personnes agnostiques, athées, sans religion et n'ayant pas renseigné l'appartenance religieuse dans les recensements de 1971, 1991 et 2011 (Source : Profile 7, Religion, Ethnicity and Irish Travellers, Census 2011, p. 12 et p. 48 et Northern Ireland Census 1971 Religion, p. 16, et Northern Ireland Census 1991 Religion, p. 18, et Table DC2118NI : Religion by sex, nisra.gov.uk)

\begin{tabular}{|l|c|c|c|}
\hline & 1971 & 1991 & 2011 (hommes + femmes) \\
\hline République d'Irlande & \multicolumn{3}{|l|}{} \\
\hline Agnostiques & n.c. & 823 & $3521(2072+1449)$ \\
\hline Athées & n.c. & 320 & $3905(2573+1332)$ \\
\hline Sans religion & 7616 & 66270 & $269811(157219+112592)$ \\
\hline Non renseignée & 5625 & 83375 & $72914(39324+33590)$ \\
\hline Irlande du Nord & 742 & $478(322+156)$ \\
\hline Agnostiques & 1200 & 470 & $1011(721+290)$ \\
\hline Athées & 512 & 59234 & $179951(100464+79487)$ \\
\hline Sans religion & \multirow{2}{|c|}{142511} & 114827 & $122252(61862+60390)$ \\
\hline Non renseignée &
\end{tabular}

Dans la République, longtemps après l'adoption de la constitution de 1937 marquée par un catholicisme traditionnel, la domination de la religion est restée omniprésente dans l'État, ses institutions et la vie de ses citoyens. Cette identité confessionnelle perdure jusque dans les années 1960 et, en 1961, à peine plus d'un millier d'individus se définissent sans religion alors que cinq fois plus ne renseignent pas l'item ${ }^{8}$.

À partir de 1971, la part des individus sans religion crôt sans interruption, passant de $0,25 \%$ à 5,8 \% de l'ensemble de la population en 2011. Au milieu des années 1990, le nombre d'individus se déclarant sans religion dépasse celui des individus n'ayant pas renseigné l'item qui s'infléchit jusqu’à la période contemporaine. Déjà en 1991, dans deux comtés, Dublin et Wicklow, un nombre supérieur d'individus sans religion avait été dénombré comparativement à ceux qui n’avaient pas divulgué leur religion. Depuis que les recensements permettent d'en savoir

8. En 1961, il y a précisément 1107 personnes se déclarant sans religion, et 5625 qui n’ont pas renseigné l’item. 
plus sur les individus se déclarant sans religion, on remarque que les hommes sont plus nombreux dans cette catégorie que les femmes, il en va de même pour l'absence d'affiliation religieuse mais de manière moins marquée.

Dans la République, les personnes se déclarant sans religion sont proportionnellement plus nombreuses chez les adultes jeunes (15-24 ans) et d'âge mûr (45-54 ans). Ils sont plus citadins que ruraux, très présents dans la capitale et ses comtés voisins ainsi que dans le Sud Ouest du pays. En 2011, les étrangers représentent près d'un tiers de l'ensemble des individus se déclarant sans religion. Trois quarts d'entre eux sont des Européens. Les Britanniques sont les plus nombreux et les Européens venus de pays où la sécularisation est en marche depuis longtemps, comme la France, l'Allemagne et la Hollande, forment un groupe d'un peu plus de 10000 personnes. Les Européens de l'Est, Polonais, Lettons et Tchèques en tête, représentent 20500 individus; parmi eux, seuls les Roumains sont très peu nombreux. Là encore, la récente immigration européenne contribue aux changements dans le paysage religieux irlandais. Cela est aussi le cas des immigrants venus d'horizons plus lointains; près de trois quarts des immigrés chinois, 7800 personnes, et 4000 immigrants venus du continent américain déclarent aussi ne pas avoir de religion.

En Irlande du Nord, la part des agnostiques, athées et personnes sans religion atteint $10 \%$ en 2011, soit son taux le plus élevé depuis que la mesure est assez précise pour les distinguer des personnes qui ne renseignent pas l'item. Les données sont en effet restées imprécises voire inexactes du fait de la situation politique des années 1960 à 1980. Il s’agit d'un taux plus élevé que dans la République et, proportionnellement, moins affecté par l'immigration. Les tendances relatives à l'âge et aux différences entre hommes et femmes sont identiques à celles observées en République.

Ce qui justifie un tel choix chez ce nombre grandissant de citoyens relève de facteurs personnels que les recensements ne traitent pas et il en va de même pour les données relatives aux individus qui ne renseignent pas l'item. On sait que leur profil reflète les tendances de ceux qui se déclarent sans religion : majoritairement des Irlandais, un grand nombre d'Européens où dominent Britanniques et Polonais, plus d'hommes que de femmes et une forte présence dans l'Est du pays.

Le refus de renseigner l'item tient sans doute à un faisceau de déterminants et soulève de nombreuses questions. S'agit-il de ne pas divulguer une donnée estimée relever de la vie intime de la personne? La formulation du questionnaire est-elle imprécise ou suscite-t-elle la confusion, qui entraîne l'absence de réponse? S'agit-il d'individus reniant la religion qu'ils avaient pratiquée jusqu'alors? Autant de questions qui demanderaient une analyse plus poussée.

On notera que les mesures de la désaffection religieuse ont fait l'objet d'une étude internationale en 2012 (WIN-Gallup International, Global Index of Religio- 
sity and Atheism) qui confirme ce que le dernier recensement irlandais soulignait, à savoir une forte progression des effectifs des non-croyants. Ce sondage représentatif indique comment les personnes se perçoivent, sans que cette perception ne soit liée à une pratique religieuse, et les données relatives à l'Irlande révèlent des taux de désaffection religieuse très supérieurs à ce que le recensement de 2011 avait établi.

Pour la catégorie sans religion telle que les recensements nationaux l'identifient, les accroissements enregistrés et les classes d'âge concernées excluent une génération spontanée de nouveaux Irlandais sans foi. On peut supposer qu'il s'agit pour le plus grand nombre d'individus préférant se définir, à un moment de leur vie, sans religion bien qu'ayant vécu jusqu'alors selon les rites et la culture d'une religion, imposée par la tradition familiale ou les institutions. On rappellera que la plupart des écoles en Irlande sont affiliées à une dénomination religieuse, et il en est de même pour les hôpitaux. En Irlande du Nord, les questionnaires des recensements tentent de cerner le contexte religieux familial de ceux qui se définissent comme sans religion. Les données rendues publiques ne permettent pas de pousser plus loin l'analyse.

\section{Conclusion}

Pour conclure, on soulignera à quel point le paysage religieux irlandais s'est transformé depuis la fin du xx siècle. En 2011, dans la République comme en Irlande du Nord, la diversité des cultes est devenue une réalité. Bien que ces pratiques ne concernent encore qu'une minorité de fidèles dans un paysage religieux encore très bi-confessionnel et qu'elles soient liées à la nouvelle composition démographique du pays, il s’agit de phénomènes inédits. L’ampleur prise par l'orthodoxie et l'islam en témoigne. Pourtant, l'essor de ces deux dénominations n'est pas exclusivement imputable à l'arrivée d'étrangers puisque pour chacune, le nombre d'Irlandais se déclarant de ces confessions a augmenté plus rapidement que celui des étrangers. La présence de ces communautés marquera la société irlandaise à moyen et long terme étant donné les effectifs et la composition des nouveaux groupes religieux.

Le bouleversement n'est pas uniquement lié à la place prise par des cultes jusqu'alors très minoritaires, il tient aussi au recul des deux religions traditionnelles. Si un niveau d'éducation plus élevé, une vie plus urbaine et des modèles de société, libérés du joug de certaines des valeurs morales prônées par les religions traditionnelles, ont contribué au recul observé, d'autres facteurs agissent par ailleurs. La composition démographique joue sur le nombre de membres protestants : ils forment un groupe plus âgé et peu renouvelé par la présence de migrants. Pour le catholicisme, de nombreux migrants d'Europe de l'Est et d'Asie 
ont contribué au maintien des effectifs, voire à une nouvelle dynamique des pratiques, un effet par ailleurs fortement affaibli par les crises du catholicisme irlandais. Enfin, les changements enregistrés depuis deux décennies révèlent une part grandissante de la population se détournant du religieux. Si le phénomène est quantitativement identifié dans les recensements, il demande une analyse qualitative plus poussée que le recueil de données statistiques ne peut documenter à ce jour. Le champ d'investigation est vaste, au niveau individuel, communautaire et institutionnel. 